\title{
An Explanation for the Bimodal Duration Distribution of Gamma-Ray Bursts: Millisecond Pulsars from Accretion-Induced Collapse
}

\author{
Insu $\mathrm{Yi}^{1}$ and Eric G. Blackman ${ }^{2}$ \\ ${ }^{1}$ Institute for Advanced Study, Olden Lane, Princeton, NJ 08540; yi@sns.ias.edu \\ ${ }^{2}$ Institute of Astronomy, Madingley Road, Cambridge CB3 OHA, England; \\ blackman@ast.cam.ac.uk
}

\begin{abstract}
Cosmological gamma-ray bursts (GRBs) could be driven by dissipation of pure electromagnetic energy (Poynting flux) extracted from rapidly rotating compact objects with strong magnetic fields. One such possibility is a young millisecond pulsar (MSP) formed from accretion-induced collapse (AIC) of a white dwarf. The combination of an efficient magnetic dynamo, likely operating during the first seconds of the initially hot and turbulent MSP interior, and the subsequent modest beaming of gamma-ray emitting outflows, would easily account for energy constraints. But the remarkable feature of such models is that they may naturally explain the hitherto unexplained bimodal distribution in GRB time durations. The two burst classes could correspond to MSPs that form spinning above and below a gravitationally unstable limit respectively. In the former case, the spin-down time scale is due to gravitational radiation emission $(<1 s)$ while the spin-down time scale of the latter is due to electromagnetic dipole emission $(\gg 1 s)$. These two time scales account for the short and long GRB durations, i.e. the observed bimodal GRB duration distribution. A natural prediction is that the short duration GRBs would be accompanied by strong gravitational radiation emission which is absent from the longer class. Both would show millisecond variabilities.
\end{abstract}

Subject headings: accretion, accretion disks - binaries: general - magnetic fields pulsars: general - stars: magnetic fields - gamma rays: bursts

\section{Introduction}

If the recently observed GRB afterglows in optical and radio are due to an expanding fireball, the total gamma-ray burst energy output is likely to be $\sim 10^{51} \Delta \Omega / 4 \pi \mathrm{erg}$ where $\Delta \Omega$ is the solid angle for the opening of the outflow from which emission occurs (e.g. Waxman et al. 1997, Dar 1997, and references therein). The fireball and its afterglow represent a generic description of how the GRB engine energy is dissipated, but there are a number of possibilities for what actually 
powers the engine. The well-known neutrino fireball model in the context of the neutron star mergers does not seem to provide sufficient energy to power the extended afterglow phase lasting $\sim$ months unless some beaming is present (Dar 1997). The required gamma-ray transparency condition on the bulk Lorentz factor of the outflow $(\Gamma>100)$ severely limits its baryon fraction (e.g. Fenimore et al. 1993). These problems have led to models in which GRBs are powered by rapidly spinning compact objects with strong magnetic fields (e.g. Usov 1992, Blackman et al. 1996, Meszaros \& Rees 1997, Paczynski 1997). In such models, power is extracted in the form of the pure electromagnetic low-frequency waves (Poynting flux) which are later converted to gamma-rays and lower frequency photons (Usov 1992, Blackman et al. 1996, Meszaros \& Rees 1997). Regardless of model details, the central engines could be expected to possess rapid rotation with spin frequencies $\sim 10^{4} s^{-1}$ and strong magnetic fields $\sim 10^{15} G$ (Usov 1992, Meszaros \& Rees 1997). Beaming should somehow be plausible for such rapidly rotating systems.

The GRB engines could either be rapidly rotating black holes surrounded by strongly magnetized tori or rapidly rotating MSPs with strong magnetic fields (Usov 1992, Meszaros \& Rees 1997). In the former scenario, the black holes could form as a consequence of the neutron star-neutron star mergers and the strong fields could be generated by dynamos in the rapidly rotating tori which are direct merger remnants. In the latter case, as first proposed by Usov (1992), the MSPs could form from the accretion induced collapse of white dwarfs. Yi and Blackman (1997) have shown that typical AIC events cannot produce a MSP with a magnetic field as strong as $\sim 10^{15} G$ if the field is solely due to the flux-frozen white dwarf fossil field. However, strong magnetic fields $\sim 10^{15} G$ could be generated by dynamo action during the hot, convective phase of the MSPs which directly follows the AIC (Duncan \& Thompson 1992).

Despite such developments, it remains a challenge to account for the vastly different time scales seen in various types of GRBs (e.g. Fishman \& Meegan 1995). Short variability time scales range $\sim 10^{-3} s$ to $\sim 0.1 s$ with a myriad of complex time profiles, but most notably, the GRB durations are known to be distributed bimodally. The short durations range from $\sim 3 \times 10^{-2} s$ to $\sim 2 s$ while the long durations range from $\sim 2 s$ up to $\sim 10^{3} s$.

The bimodal distribution suggests that something discrete distinguishes the two classes. An interesting advantage of the AIC/MSP scenario over the black hole scenario in this respect is that two classes of pulsars naturally form (Usov 1992), and can therefore explain the bimodality. When a MSP rotates with spin frequency larger than a certain critical frequency (e.g. Friedman 1983), non-axisymmetric secular instability drives the pulsar into non-axisymmetric configuration with non-zero quadrupole moment. In this case, the spin-down of the pulsar occurs on a time scale

$$
\tau_{g w}=I_{*} \Omega_{*}^{2} / 2 L_{g w} \sim 3 \times 10^{-3} \epsilon^{-2} I_{*, 45} \Omega_{*, 4}^{-4} \quad s
$$

where $I_{*}=I_{*, 45} 10^{45} \mathrm{gcm}^{2}$ is the MSP moment of inertia $\epsilon=\delta R_{*} / R_{*}$ is the ellipticity of non-axisymmetrically deformed pulsar with radius $R_{*}$ and perturbation $\delta R_{*}, \Omega_{*}=\Omega_{*, 4} 10^{4} s^{-1}$ is the MSP spin frequency, and we have made use of the gravitational wave energy loss rate

$$
L_{g w}=32 G \epsilon^{2} I_{*}^{2} \Omega_{*}^{6} / 5 c^{5} .
$$


On the other hand, the spin-down due to the electromagnetic dipole radiation gives a time scale

$$
\tau_{e m} \sim I_{*} \Omega_{*}^{2} / 2 L_{e m} \sim 3 \times 10^{2} I_{*, 45} R_{*, 6}^{-6} B_{*, 15}^{-2} \Omega_{*, 4}^{-2} \quad s
$$

where $R_{*}=R_{*, 6} 10^{6} \mathrm{~cm}$ is the MSP radius, $B_{*}=B_{*, 15} 10^{15} \mathrm{G}$ is the MSP dipole magnetic field and we have assumed the simple electromagnetic dipole energy loss rate

$$
L_{e m}=2 \mu_{*}^{2} \Omega_{*}^{4} / 3 c^{3}
$$

where $\mu_{*}=B_{*} R_{*}^{3}$ is the electromagnetic dipole moment. Usov (1992) pointed out these two natural time scales, and here we explore how they may account for the bimodal distribution of GRBs.

We consider the AIC scenario and carefully examine how plausible physical conditions may facilitate an explanation for bimodality. It turns out that it is necessary to consider hot neutron star conditions under which high neutrino viscosity and convection are important as noted by Duncan and Thompson (1992). We then discuss possible implications of the AIC-MSP interpretation of the bimodal duration distribution.

\section{Pulsars near Critical Rotation}

When a white dwarf reaches the critical Chandrasekhar mass $\sim 1.4 M_{\odot}$ through mass accretion, the white dwarf can collapse to a neutron star. The probable mass accretion rate leading to AIC is $\dot{M} \gtrsim 3 \times 10^{18} \mathrm{~g} / \mathrm{s}$ (e.g. Livio \& Truran 1992). Even at these high mass accretion rates, the mass accretion should be sustained for $>10^{6} \mathrm{yr}$. Such constraints generally disfavor dwarf binary systems in which the secondary mass donors are dwarf stars. The white dwarf magnetic fields interact with accretion flows and directly affect its subsequent spin evolution. But given the long accretion time scales, the initial spins of the white dwarfs have little effect on the final outcome of the pre-collapse white dwarf (Yi \& Blackman 1997). For a white dwarf with moment of inertia $I_{w d}=10^{51} \mathrm{gcm}^{2}$ and radius $R_{w d}=10^{9} \mathrm{~cm}$, the magnetic field $B_{w d}$ and spin frequency $\Omega_{w d}$ are related to the post-AIC pulsar spin frequency and magnetic field by $\Omega_{w d}=\Omega_{*}\left(I_{*} / I_{w d}\right)$ and $B_{w d}=B_{*}\left(R_{*} / R_{w d}\right)^{2}$ where $I_{*}=10^{45} \mathrm{gcm}^{2}$ and $R_{*}=10^{6} \mathrm{~cm}$ are the moment of inertia and the radius of the MSP. In order to create a $\operatorname{MSP}\left(\Omega_{*} \sim 10^{4} s^{-1}\right)$ with $B_{*} \sim 10^{15} G$ by flux-freezing, the pre-collapse white dwarf must have $\Omega_{w d} \sim 10^{-2} s$ and $B_{w d} \sim 10^{9} G$. Such strong white dwarf

fields have not been observed. However, even when such a field exists, Yi \& Blackman (1997) have shown that such pre-AIC magnetized accretion is not compatible with such a white dwarf due to efficient magnetic braking and spin-down during pre-AIC magnetized accretion phase. They find that the most likely AIC-produced MSP parameters satisfy

$$
\Omega_{*} \sim 10^{4} B_{*, 11}^{-4 / 5} s^{-1}
$$


where $B_{*, 11}=B_{*} / 10^{11} G$.

Duncan \& Thopmson (1992) suggested that the hot MSP which is likely to form from AIC is a favorable site for an efficient dynamo of $\alpha \omega$ type due to the vigorous convection driven by a large neutrino flux which is the direct outcome of the white dwarf to neutron star collapse. During the first seconds, the large neutrino flux drives convection. When the convection is significant, the efficiency of the dynamo could be roughly estimated by the Rossby number

$$
N_{R}=P_{*} / \tau_{\text {conv }}
$$

where $P_{*}=2 \pi / \Omega_{*}$ is the MSP spin period and $\tau_{\text {conv }}$ is the convective overturn time scale at the base of the convection zone. If $N_{R}$ is of order unity or less in a turbulent medium, the amplification of field by helical motion is not suppressed by turbulent diffusion and an efficient dynamo results. During the hot neutrino phase, the neutrino viscosity greatly exceeds the kinematic shear viscosity appropriate to cool neutron stars, $\nu \sim 1-100 \mathrm{~cm}^{2} \mathrm{~s}^{-1}$. Following Duncan and Thompson (1992), the convective overturn time is roughly given by $\tau_{\text {conv }} \sim 10^{-3} F_{39}^{-1 / 3} s$ where $F_{39}$ is the convective neutrino heat flux in units of $10^{39} \mathrm{erg} / \mathrm{s} / \mathrm{cm}^{2}$.

The uncertainty associated with the required spin rate for an efficient dynamo action, $\Omega_{\text {dynamo }} \sim 2 \pi \tau_{\text {conv }}^{-1} \lesssim 6 \times 10^{3} s^{-1}$ is largely due to the uncertainty in the effectiveness of the dynamo when $N_{R} \sim 1$ and the uncertainty in the convective overturn time scale. The increase of $N_{R}$ from $\sim 1$ by an order of magnitude seems to quench the build-up of the strong fields (Simon 1990). Nevertheless, Duncan \& Thompson (1992) show that a dynamo generated large scale dipole field $\sim 10^{15} G$ can result. Given the possibility of some dynamo action at $N_{R} \gtrsim 1$, the dynamo-generated field may still exist for $\Omega_{*} \lesssim \Omega_{\text {dynamo }}$.

The secular instability driven by the gravitational wave emission and damped by the shear viscosity perturbs the axi-symmetric star with a non-axisymmetric perturbation of the form (e.g. Wagoner 1984)

$$
\delta R_{*}=\Sigma_{l, m} \delta R_{l m} Y_{l}^{m}(\theta, \phi) \exp (i \omega t)
$$

where the instability sets in via a mode with $\omega=m \Omega_{*}$ (Friedman 1983). There are in principle two ways in which a gravitationally unstable star can radiate away energy. It can 1) spin down, or 2) spin up, but lower its moment of inertia accordingly. The former is relevant when the fast spinning neutron star is approximated as a classical Maclaurin spheroid. This approximation amounts to the condition that the neutron star equation of state is sufficiently stiff (e.g. Chandrasekhar 1969, Yi \& Blackman 1997) and that the internal vorticity of the star in the rotating ellipsoidal pattern frame, exceeds twice the pattern speed measured in the inertial frame (Lai \& Shapiro 1995). Evolution 2) corresponds to a Jacobi to Maclaurin transition, and would occur when the inequality, described above, is reversed. Track 2) also requires the gravitational perturbation time to be slower than the gravitational radiation time, otherwise the star could not adjust its radius quickly enough to change its moment of inertia as required. Though the initial conditions determine which evolution the star will follow, we will see that the growth time turns out to be of 
order the gravitational radiation time. We therefore suspect that spin-down evolution is generally more likely, and we focus on the Maclaurin evolution.

The perturbation growth time scale for the Maclaurin spheroid is

$$
\tau_{g r}=\frac{(m-1)[(2 m+1) ! !]^{2}}{(m+1)(m+2)\left(1-e^{2}\right)^{1 / 2}}\left(\frac{c}{R_{*} \omega}\right)^{2 m+1}\left(\frac{\omega+(m-1) \Omega_{*}}{2 \pi G \rho_{*}}\right)
$$

where $\rho_{*}$ is the stellar density and $e$ is the eccentricity of the MSP when the instability sets in (Comins 1979, Friedman 1983, Lindblom 1986). For a neutron star $t_{d y n}^{-1}=\sqrt{\pi G \rho_{*}} \approx 10^{4} s^{-1}$. Since $\omega \sim t_{d y n}^{-1}$ using the values of $e$ for each model $l=m$, the growth time scale becomes (Friedman $1983)$

$$
\tau_{g r} \approx a t_{d y n}\left(c / R_{*} \omega\right)^{2 m+1}
$$

where $a \approx 10,10^{3}, 10^{5}, 10^{7}$ for $m=2,3,4,5$ respectively. Therefore, $\tau_{g r} \approx 1,10^{3}, 10^{6}, 10^{9} s$ for $m=2,3,4,5$. The major uncertainty comes from the sensitive dependence of $\tau_{g r}$ on $\omega$. The $l \neq m$ modes become unstable only after $l=m$ bar modes become unstable so they are irrelevant for our discussions.

The large neutrino viscosity of hot young pulsars leads to much shorter damping time scales compared to those of cold neutron stars. The viscous damping time scale is (Comins 1979, Friedman 1983, Lindblom 1986)

$$
\tau_{v i s} \approx \frac{1}{(m-1)(2 m+1)} \frac{R_{*}^{2}}{\nu}
$$

In ordinary cold neutron stars, $\nu \sim 1-100 \mathrm{~cm}^{2} / \mathrm{s}$ and hence the viscous damping time scale $\tau_{v i s} \approx\left[\left(10^{10}-10^{12}\right) /(m-1)(2 m+1)\right] s$. All modes with $m \leq 4$ are in principle excited. The critical frequency is then set by $m=4$ mode as the highest $m$ mode gives the lowest critical frequency. However, right after AIC, when MSP is hot, the kinematic shear viscosity is determined by the neutrino viscosity which is close to $\sim$ a few $\times 10^{9} \mathrm{~cm}^{2} \mathrm{~s}^{-1}$ for $\rho_{*}=10^{15} \mathrm{~g} / \mathrm{cm}^{3}$ and the MSP temperature of $\sim 10^{10} K$ (Duncan \& Thompson 1992). The corresponding viscous damping time scale is $\tau_{v i s} \approx\left[10^{3} /(m-1)(2 m+1)\right] s$ or $\tau_{v i s} \approx 2 \times 10^{2}, 70,40,20 s$ for $m=2,3,4,5$, respectively. Therefore, for this high viscosity, all modes with $m>2$ are rapidly damped but the $l=m=2$ mode grows. The critical frequency for the secular instability is then simply determined by the $l=m=2$ instability. Despite various uncertainties in estimating the time scales, $\tau_{g r}<\tau_{v i s}$ is likely to be satisfied by the $l=m=2$ mode. This implies that the non-axisymmetric perturbation occurs at a frequency closest to the dynamical break-up frequency. In cool neutron stars (with low viscosity), the critical frequency for the secular instability is determined by higher $l=m$ modes and as a consequence the critical frequency is substantially lower than that associated with the $l=m=2$ mode.

For $l=m=2$ mode in the Maclaurin spheroids, the critical frequency is given by (Chandrasekhar 1969)

$$
\Omega_{c r i t}=0.612 t_{d y n}^{-1}
$$


at which point the eccentricity of the spheroid $e=0.813$. The effect of the relativistic corrections is at the level of $\sim 10 \%$ if $G M / R c^{2} \sim 0.3$ (Balbinski \& Schutz 1982). For pulsars with $\Omega_{*}>\Omega_{c r i t}$, the $l=m=2$ model leads to perturbed ellipsoid and develops a non-zero quadrupole moment. The spin-down during this stage is roughly described by

$$
\frac{d \Omega_{*}}{d t}=-s \Omega_{c r i t} \tau_{g r}^{-1} \frac{M_{*}\left(\delta R_{*}\right)^{2}}{I_{*}},
$$

where $s$ is a constant of order unity. The $l=m=2$ mode grows quickly, building up to $\delta R_{*} \lesssim R_{*}$. Further growth is limited by nonlinear effects.

The loss of energy by gravitational radiation from an unstable Maclaurin spheroid proceeds through spin down. The gravitational radiation depletes energy and angular momentum while $\delta R_{*}$ remains nearly constant. Eventually, the gravitational instability growth time scale gets longer as spin-down continues until $\tau_{g r}>\tau_{v i s}$ occurs for $l=m=2$. For $s \sim 1, I_{*} \sim$ constant, $\delta R_{*} \sim R_{*}$ and $\Omega_{*} \sim \Omega_{\text {crit }}$, the spin-down time scale $\tau_{\text {down }} \sim \tau_{\text {gr }}$.

In general, when the gravitational instability growth time scale is of order the gravitational radiation drain time scale the star does not have time to change its moment of inertia, so gravitational radiation proceeds through spin-down. This is a likely state for a highly viscous nascent neutron star. This also means that the electromagnetic luminosity is depleted on the spin-down time scale, enabling the AIC-MSP model to account for the bimodality of GRB durations, as addressed in the next section. Note that if the young NS initially were somehow to follow the Jacobi track 2) as discussed above, it would instead spin up, and would not naturally lead to a bimodal distribution. However, the gravitational radiation from these two paths are very distinct, and will be measurable (Lai \& Shapiro 1995). Either way, the signature of GRB from AIC-MSP should be testable.

\section{Bimodal Distribution}

Based on the discussion of the previous section, we classify three classes of hot MSPs relating to GRB engines. This essentially involves three rotational frequencies $\Omega_{*}, \Omega_{\text {dynamo }}, \Omega_{\text {crit }}$ (cf. Blackman et al. 1996). If MSPs are formed from AIC of magnetized white dwarfs, the initial MSP frequencies are determined by the pre-collapse white dwarf magnetic field strengths (Yi \& Blackman 1997).

Since we assume that $\Omega_{\text {crit }}>\Omega_{\text {dynamo }}$, the three classes are 1) supercritical rotators with strong fields (SPS) with $\left.\Omega_{*}>\Omega_{\text {crit }}>\Omega_{\text {dynamo }}, 2\right)$ subcritical rotators with strong fields (SBS) with $\Omega_{\text {crit }}>\Omega_{*}>\Omega_{\text {dynamo }}$, and 3) subcritical rotators without dynamo action (SBW) with $\Omega_{\text {crit }}>\Omega_{\text {dynamo }}>\Omega_{*}$. The last class lacks strong magnetic fields due to the absence of dynamo action. Since the three classes have very similar rotational frequencies, they likely originate from 
very similar pre-collapse initial conditions of the accreting white dwarf phase (Yi \& Blackman 1997). Therefore, for an arbitrary distribution of initial white dwarf conditions (cf. eq. 2-1), the numbers of the three classes of objects are expected to be similar.

Because the classes are distinguished by small differences in their spin frequencies, the electromagnetic dipole luminosity differs in each class. In the two classes, SPS and SBS, the efficient dynamo action results in $B_{*} \sim 10^{15} \mathrm{G}$, and the dipole luminosity gives

$$
L_{e m} \sim 2 \times 10^{50} R_{*, 6}^{6} B_{*, 15}^{2} \Omega_{*, 4}^{4} \mathrm{erg} / \mathrm{s}
$$

We expect that SPS's $L_{e m}\left(\propto \Omega_{*}^{4}\right)$ is slightly larger than SBS's due to slightly larger $\Omega_{*}$ for SPS. This is apparently consistent with the observed luminosity difference between the long bursts and short bursts (e.g. Fishman \& Meegan 1995). Despite the similar luminosities, the luminosity evolution time scales are very different. In SPS, the spin-down, and hence the luminosity decrease, occurs on a time scale $\sim \tau_{g r} \sim \tau_{g w} \lesssim 1 s$ whereas in SBS, the spin-down and luminosity decrease occur on a time scale $\sim \tau_{e m} \sim 10^{2} s$. We therefore naturally relate SPS to the short bursts and SBS to long bursts. The third class, SBWs, are expected to have luminosities (cf. eq. 2-1)

$$
L_{e m} \sim 10^{44} R_{*, 6}^{6} \Omega_{*, 4}^{3 / 2} \mathrm{erg} / \mathrm{s}
$$

based on the AIC scenario of Yi \& Blackman (1997). Therefore, unless there is strong beaming, the third class is not expected to give rise to cosmological GRBs.

If the critical frequency $\Omega_{\text {crit }}$ were much lower than the assumed value (which can happen if the shear viscosity is much lower), the non-axisymmetric instability could develop at lower $\Omega_{*}$ 's corresponding to higher $l=m>2$. However, even in this case, the growth time scale $\tau_{g r}>10^{3} \mathrm{~s}$ for $l=m>2$ bar modes are too long to be of any relevance for AIC produced, hot MSPs. During the vigorous convection phase, which lasts $\sim 30 s$, the $\tau_{e m}$ is shorter than $\tau_{g r}$ for $l=m>2$, which implies that the non-axisymmetry does not develop before the spin-down occurs through electromagnetic phase. Such bursts would resemble those of the SBS class.

If we relate the electromagnetic dipole power to the observed gamma-ray luminosity assuming $\xi$ as the efficiency to convert the pure electromagnetic power to gamma-ray luminosity and the physical solid angle of the outflow is $\Delta \Omega$, we get $\Delta \Omega / 4 \pi=\xi L_{e m} / L_{\gamma}$ where $L_{\gamma}$ is the observed isotropic gamma-ray luminosity. Therefore, for $L_{\gamma} \sim 10^{51} \mathrm{erg} / \mathrm{s}$ and $L_{e m} \sim 2 \times 10^{50} \mathrm{erg} / \mathrm{s}$, we get $\Delta \Omega / 4 \pi \sim 0.2 \times \xi$. For a low efficiency $\xi \sim 10^{-3}$, the required beam size is as small as $\Delta \Omega \sim 10^{-3}$. If the outflow's bulk Lorentz factor is $\Gamma$, the physical beam size need not be smaller than that associated with the relativistic beaming unless $\Gamma \lesssim 30$. This bulk Lorentz factor is below the required value of $\Gamma$ for the gamma-ray transparency (e.g. Rees 1997), so the jet needs to be only modestly beamed.

\section{Discussion}


The observed bimodal GRB duration distribution has been a mystery. We have suggested that well-known time scales which are plausible for the AIC-MSP scenario may account for the bimodality. Pulsars formed above the gravitationally unstable limit deplete their energy through gravitational radiation, thereby limiting the amount of energy available for GRB, and providing the short class. The long class comes from the gravitationally stable rotators.

Note that this approach to bimodality focuses on the GRB engine rather than on the subsequent nature of how the engine energy is dissipated. The latter approach is taken by Sari and Piran (1995) who suggest that in the context of a (spherical) fireball model, bimodality may arise from differing roles of Newtonian vs. relativistic reverse shocks in energy dissipation. Associating the bimodality with the engine rather than the dissipation assumes that the dissipation mechanism and the efficiency of energy extraction into gamma-rays occurs statistically in the same way for the two burst populations. However, we predict a higher luminosity for the shorter bursts, because the unstable rotators would have the larger spin and thus the larger electromagnetic emission before slowing down rapidly by gravitational radiation.

An observational signature for associating bimodality directly with an AIC-MSP source engine would come from the fact that our shorter SPS class of GRBs are accompanied by gravitational radiation which is absent from the longer SBS class. Provided that young gravitationally radiating AIC-MSP deplete their rotational energy through spin-down as described herein, the detection of gravitational radiation from short GRBs well in excess of that from long GRBs would give strong support to the AIC/MSP engine model (Blackman et al. 1996). The gravitational radiation signature for various evolutionary tracks of secularly unstable AIC pulsars are unique and measurable (Lai \& Shapiro 1995). In general, gravitational radiation may provide unique signatures to source engines and be the most promising means of observationally distinguishing engine models.

If the central engine for GRBs is indeed a rapidly rotating MSP, the stellar rotation itself may be the source of variability. The typical MSPs' rotational frequency could naturally give rise to rapid variabilities on time scales of $\sim 10^{-2}-10^{-3} s$. In order for the stellar rotation to show up as variability, it is required that the magnetic dipole axis is misaligned with the rotational axis. For large misalignment angles, the amplitudes of variable fluxes are expected to be large. If this simple mechanism is indeed responsible for the variability, duration and variability time scale are not expected to be correlated. Given the complex burst time profiles (Fishman \& Meegan 1995), it is interesting that the shortest variability time scale is comparable to the shortest GRB duration. In the MSP scenario, this is naturally explained as the MSP spin-down time scale can be comparable to MSP spin period. Note also the fact that the GRB engine in this model would remain a stable pulsar long after the gamma-ray emission.

If GRBs are well collimated, the long term evolution of the beam is likely to be influenced by the precession of the MSP jet axis (Blackman et al. 1996). Since AIC occurs in a binary system (likely to be a close binary system), in which the secondary star is a main sequence star with mass 
$M_{2} \sim 0.5 M_{\odot}$, the expected precession time scale due to the Lense-Thirring precession is (e.g. Martin \& Rees 1979)

$$
P_{L T} \sim \frac{c^{2}}{2(2 \pi)^{2 / 3} G^{2 / 3}} \frac{\left(M_{*}+M_{2}\right)^{4 / 3}}{M_{*} M_{2}} P_{o r b}
$$

where $P_{\text {orb }}$ is the orbital period of the pulsar system and $M_{*} \sim 1.4 M_{\odot}$. $P_{\text {orb }} \sim 1 h r$ gives $P_{L T} \sim$ a few yrs. Therefore, if the gamma-ray emission and especially afterglow (on a time scale $\sim$ yr) occurs within a beam, the precession could give a long term evolution trend.

The rate of AIC remains uncertain. If the AIC rate is comparable to the local supernova rate, i.e. $1-10^{-2} \mathrm{yr}^{-1}$ per galaxy (Blair 1989), the observed gamma-ray burst rate of $\sim 10^{-6} \mathrm{yr}^{-1}$ per galaxy is amply explained by the AIC model if $\Delta \Omega \sim 10^{-5}-10^{-3}$ which is largely consistent with the luminosity requirement if $\xi \sim 10^{-3}$.

The general features of an afterglow from the AIC-MSP scenario may not be drastically different from that of any other scenario, since an afterglow represents the dissipation of GRB energy once it has been produced. Our focus on the AIC-MSP model is an attempt to address the physics of the GRB engine source. It is important to distinguish the engine physics from that of the fireball and afterglow in the same way that the physics of an evolving supernova remnant needs to be distinguished from the physics of its engine core collapse. Here we have investigated the possibility that the bimodal distribution may actually be a signature for an AIC-MSP GRB engine.

I. Y. acknowledges support from SUAM Foundation.

\section{REFERENCES}

Balbinski, E. \& Schutz, B. F. 1982, MNRAS, 200, 43

Blackman, E. G., Yi, I., \& Field, G. B. 1996, ApJ, 473, L79

Blair, D. 1989, in Gravitational Wave Data Analysis ed. B. F. Schutz (Dordrecht: Kluwer)

Chandrasekhar, S. 1969, Ellipsoidal Figures of Equilibrium (New Haven: Yale University Press)

Comins, N. 1979, MNRAS, 189, 233

Dar, A. 1997, preprint astro-ph/9709231)

Duncan, R. C. \& Thompson, C. 1992, ApJ, 392, L9

Fenimore, E. E., Epstein, R. I., \& Ho, C. 1993, A\&AS, 97, 59

Fishman, G. J. \& Meegan, C. A. 1995, ARA\&A, 33, 415

Friedman, J. L. 1983, PRL, 51, 11

Lindblom, L. 1986, ApJ, 303, 146 
Lai, D. \& Shapiro, S.L., ApJ, 442, 559

Livio, M. \& Truran, J. W. 1992, ApJ, 389, 695

Martin, P.G., \& Rees, M.J., MNRAS, 1979, 189, 19

Meszaros, P. \& Rees, M. J. 1997, ApJ, 482, L29

Pazcynski, B. 1997, preprint (astro-ph/9710086)

Rees, M.J., preprint astro-ph/9701162

Sari, R. \& Piran, T. 1995, ApJ, 455, L143

Simon, T. 1990, ApJ, 359, L11

Usov, V. V. 1992, Nature, 357, 452

Wagoner, R. V. 1984, ApJ, 278, 345

Waxman, E. 1997, preprint (astro-ph/9709199)

Yi, I. \& Blackman, E. G. 1997, ApJ, 482, 383 